\title{
Uso de leguminosas na fitorremediação de solo contaminado com sulfentrazone ${ }^{1}$
}

\author{
João Carlos Madalão ${ }^{2}$, Fábio Ribeiro Pires ${ }^{3}$, \\ Kristhiano Chagas $^{3}$, Alberto Cargnelutti Filho ${ }^{4}$, Sergio Oliveira Procópio ${ }^{5}$
}

\begin{abstract}
Use of leguminous plants for phytoremediation of soil contaminated with sulfentrazone

Residual sulfentrazone may remain in the soil for around two years, being a source of groundwater contamination and preventing the growth of susceptible plants. So, phytoremediation may represent an option for decontaminating areas which were highly affected by applications of this herbicide. This study aimed at evaluating the potential of Crotalaria juncea, Canavalia ensiformis, Cajanus cajan and Cajanus cajan (dwarf) for remediation of soil contaminated with sulfentrazone, using millet (Pennisetum glaucum) as a bioindicator plant. The experiment was installed in a greenhouse and treatments consisted of the four species already mentioned, plus a control, and four sulfentrazone doses $\left(0 \mathrm{~g}\right.$ a.i. ha $\mathrm{h}^{-1}, 200 \mathrm{~g}$ a.i. ha $\mathrm{ha}^{-1}, 400 \mathrm{~g}$ a.i. ha $\mathrm{ha}^{-1}$ and $800 \mathrm{~g}$ a.i. ha $\mathrm{h}^{-1}$ ). Plants height, phytotoxicity to sulfentrazone and fresh and dry matter were evaluated. When P. glaucum was grown after $C$. juncea, the fresh and dry matter, as well as plant height, were higher, while the phytotoxicity symptoms were less evident. C. juncea showed the best results for phytoremediation of soils contaminated with sulfentrazone.
\end{abstract}

KEY-WORDS: Crotalaria juncea L.; soil decontamination; residual herbicide.

\section{INTRODUÇÃO}

A utilização de herbicidas é prática comum na atividade agropecuária e consiste em importante ferramenta para se alcançar elevadas produções em áreas extensas (Pires et al. 2008, Procópio et al. 2008). Todavia, o emprego de moléculas que apresentam longo período residual no solo pode resultar na impossibilidade de uso da área por espécies de

\section{RESUMO}

Resíduos de sulfentrazone podem permanecer no solo por cerca de dois anos, oferecendo risco de contaminação a lençóis freáticos e impossibilitando o cultivo de espécies susceptíveis. Diante disto, a fitorremediação pode representar uma opção para a descontaminação de áreas que sofreram com intensas aplicações deste herbicida. O presente trabalho avaliou o potencial remediador das espécies Crotalaria juncea, Canavalia ensiformis, Cajanus cajan e Cajanus cajan (anão), com relação ao herbicida sulfentrazone, utilizando-se o milheto (Pennisetum glaucum) como planta bioindicadora. $\mathrm{O}$ experimento foi instalado em casa-de-vegetação e os tratamentos compostos pela combinação entre as quatro espécies citadas, além da testemunha, e quatro doses de sulfentrazone $\left(0 \mathrm{~g}\right.$ i.a. ha ${ }^{-1}$, 200 g i.a. ha-1, 400 g i.a. ha ${ }^{-1}$ e 800 g i.a. ha $\left.{ }^{-1}\right)$. Foram avaliadas a altura de plantas, fitotoxicidade ao sulfentrazone e biomassa fresca e seca da parte aérea. Quando P. glaucum foi cultivado após $C$. juncea, apresentou maior ganho em biomassa e maior altura, e os sintomas de fitotoxicidade foram menos acentuados. C. juncea apresentou a maior capacidade de fitorremediar solos contaminados com sulfentrazone.

PALAVRAS-CHAVE: Crotalaria juncea L.; descontaminação do solo; residual de herbicidas.

interesse agrícola, susceptíveis ao herbicida em questão (Procópio et al. 2004, Santos et al. 2004a e 2004b).

O sulfentrazone, recomendado para uso em pré-emergência na cultura da cana-de-açúcar, destaca-se como herbicida de larga utilização e que apresenta longo período residual no solo (Vivian et al. 2006, Blanco et al. 2010, Melo et al. 2010, Monquero et al. 2010).

1. Trabalho recebido em mar./2012 e aceito para publicação em out./2012 (n registro: PAT 17494).

2. Universidade Federal de Viçosa (UFV), Departamento de Fitotecnia, Viçosa, MG, Brasil. E-mail: joaocarlosagr@hotmail.com.

3. Universidade Federal do Espírito Santo (UFES), Centro Universitário Norte do Espírito Santo, Departamento de Ciências Agrárias e Biológicas, São Mateus, ES, Brasil.E-mails: fabiopires@ceunes.ufes.br, kristhianoc@gmail.com.

4. Universidade Federal de Santa Maria (UFSM), Centro de Ciências Rurais, Departamento de Fitotecnia, Santa Maria, RS,

Brasil. E-mail: alberto.cargnelutti.filho@gmail.com.

5. Embrapa Soja, Londrina, PR, Brasil.E-mail: procopio.so@gmail.com. 
A persistência desse herbicida, aplicado à cultura da soja, foi determinada em 376 dias, após a aplicação de $0,6 \mathrm{~kg}$ do ingrediente ativo (i.a.) ha $\mathrm{ha}^{-1}$. Já para a dose de $1,20 \mathrm{~kg}$ i.a. ha ${ }^{-1}$, não foi possível determinar o final da persistência, pois, mesmo na última época amostrada (539 DAT), a beterraba, espécie utilizada como bioindicadora, mostrou-se sensível ao herbicida (Blanco \& Velini 2005). A persistência do sulfentrazone, aplicado à cultura da cana-de-açúcar, foi quantificada em 601 dias e 704 dias, após a aplicação das doses de $0,6 \mathrm{~kg}$ i.a. ha ${ }^{-1}$ e 1,20 kg i.a. ha- ${ }^{-1}$, respectivamente (Blanco et al. 2010).

Considerando-se os dados de persistência acima e, ainda, que a cana-de-açúcar ocupou área de cerca de 8,5 milhões de hectares, na safra 2012/2013 (Conab 2012), com previsão de 14 milhões de hectares para 2020 (Unica 2010), o sulfentrazone pode constituir-se em fonte de contaminação de aquíferos e extensas áreas, principalmente como resultado de aplicações sequenciais ao longo dos anos. Dentre as espécies altamente sensíveis ao sulfentrazone, encontra-se o Pennisetum glaucum, cuja susceptibilidade foi comprovada por Dan et al. (2011), ao analisarem a atividade residual deste herbicida, aplicado em pré-emergência à cultura da soja, sobre o milheto cultivado em sucessão.

Diante disso, é necessário o emprego de técnicas que acelerem a retirada desse composto do solo, promovendo sua descontaminação. Além do benefício ambiental, a fitorremediação resultaria, adicionalmente, na possibilidade de se introduzir culturas econômicas susceptíveis, em sucessão à cana-de-açúcar, incrementando a renda, mesmo durante a renovação do canavial, o que tornaria esta operação mais sustentável.

A fitorremediação é uma alternativa ambientalmente aceita para a despoluição do solo e água contaminados com compostos orgânicos e inorgânicos. Em razão disto, é crescente o número de pesquisas envolvendo a fitorremediação. Todavia, tais estudos são ainda incipientes e requerem muitas experimentações sob condições edafoclimáticas tropicais, para identificação de espécies capazes de atuar em programas de fitorremediação, à semelhança do que já é realizado na Europa, EUA, Nova Zelândia e Austrália (Pires et al. 2009).

Recentes pesquisas têm difundido a utilização de plantas na remediação de pesticidas, e algumas apontam a eficiência de plantas na remediação de herbicidas como o tebuthiuron (Pires et al. 2005a, 2005b, 2006 e 2008), trifloxysulfuron sodium (Santos et al. 2004a) e picloram (Procópio et al. 2008), no Brasil, e atrazine (Arthur et al. 2000), simazine (Wilson et al. 2000) e metolachlor (Anderson \& Coats 1995), em condições de clima temperado. Em relação ao sulfentrazone, Belo et al. (2011) apontam as espécies Helianthus annus, Canavalia ensiformis, Dolichos lab lab e Arachis hypogaea como indicadas para a fitorremediação deste herbicida. Em todos estes trabalhos, foram realizados ensaios biológicos, e as variáveis biométricas relacionadas ao crescimento das plantas, como altura, biomassa, área foliar e sintomas de fitotoxicidade, mostraram-se eficientes em evidenciar as respostas biológicas esperadas diante da presença de moléculas herbicidas no solo.

O mecanismo responsável pela remediação, em cada uma dessas moléculas, ainda não está bem elucidado, mas, no caso da fitorremediação de herbicidas (particularmente do sulfentrazone), a fitodegradação é o mecanismo mais desejável, considerando-se a possibilidade de completa mineralização do contaminante por esta via. Com isto, as plantas podem ser mantidas na mesma área em que ocorreu a fitorremediação, resultando em melhorias para o solo, como no caso dos adubos verdes e outras plantas de cobertura (Procópio et al. 2007).

Em trabalho realizado com a finalidade de selecionar espécies tolerantes ao sulfentrazone, para fitorremediação, dentre as 25 espécies testadas, Madalão (2011) identificou, como as mais promissoras, a crotalária júncea (Crotalaria juncea), feijão de porco (Canavalia ensiformis), feijão guandu (Cajanus cajan) e feijão guandu anão (Cajanus cajan anão). Assim, o presente trabalho objetivou avaliar o potencial remediador destas quatro espécies, em relação ao herbicida sulfentrazone, utilizando-se o milheto (Pennisetum glaucum) como planta bioindicadora.

\section{MATERIAL E MÉTODOS}

O experimento foi instalado em casa-de-vegetação localizada em São Mateus (ES) (1843'S, $39^{\circ} 51^{\prime} \mathrm{W}$ e altitude de $39,0 \mathrm{~m}$ ), de janeiro a junho de 2010, utilizando-se vasos com capacidade para $10,0 \mathrm{dm}^{3}$, sem furos e preenchidos com Argissolo Amarelo (LA) coeso (Embrapa 2006), coletado à profundidade de $0,0-20,0 \mathrm{~cm}$ e peneirado em malha de 4,0 $\mathrm{mm}$.

Foi coletada uma amostra desse solo, a qual, em seguida, foi seca ao ar e peneirada em malha de 
2,0 mm, obtendo-se, assim, amostra de terra fina para posteriores análises químicas. Foram determinados o $\mathrm{pH}$ em $\mathrm{H}_{2} \mathrm{O}(5,4)$, $\mathrm{P}$ e $\mathrm{K}^{+}$extraíveis em Mehlich $^{-1}$ (3,0 $\mathrm{mg} \mathrm{dm}^{-3}$ e $0,18 \mathrm{cmol}_{\mathrm{c}} \mathrm{dm}^{-3}$, respectivamente), teores de $\mathrm{Ca}^{2+}, \mathrm{Mg}^{2+}$ e $\mathrm{Al}^{3+}$ trocáveis extraídos em KCl $1 \mathrm{~mol} \mathrm{~L}^{-1}\left(1,8 \mathrm{cmol}_{\mathrm{c}} \mathrm{dm}^{-3}, 1,0 \mathrm{cmol}_{\mathrm{c}} \mathrm{dm}^{-3}\right.$ e $0,1 \mathrm{cmol}_{\mathrm{c}} \mathrm{dm}^{-3}$, respectivamente) e Carbono $\left(22,8 \mathrm{~g} \mathrm{~kg}^{-1}\right)$ (Embrapa 1999). Com os valores obtidos nas análises do solo, calculou-se a CTC efetiva $\left(3,08 \mathrm{cmol}_{\mathrm{c}} \mathrm{dm}^{-3}\right)$, a soma de bases trocáveis (SB) $\left(2,98 \mathrm{cmol}_{\mathrm{c}} \mathrm{dm}^{-3}\right)$, a percentagem de saturação por alumínio (m) $(3,24 \%)$ e a percentagem de saturação por bases (V) (53,9\%) (Embrapa 1999). A textura do solo foi determinada conforme Embrapa (1997), obtendo-se os seguintes valores: areia $=750 \mathrm{~g} \mathrm{~kg}^{-1}$, silte $=30 \mathrm{~g} \mathrm{~kg}^{-1}$ e argila $=220 \mathrm{~g} \mathrm{~kg}^{-1}$.

Os tratamentos foram compostos pela combinação entre as espécies crotalária júncea ( $C$. juncea), feijão de porco (C. ensiformis), feijão guandu (C. cajan) e feijão guandu anão (C. cajan anão), mais um tratamento sem cultivo (testemunha), e quatro doses do sulfentrazone ( 0 g i.a. ha ${ }^{-1}, 200$ g i.a. ha-1, 400 g i.a. ha ${ }^{-1}$ e 800 g i.a. ha $\left.{ }^{-1}\right)$. Utilizou-se delineamento em blocos casualizados, em esquema fatorial $5 \times 4$, com quatro repetições.

Após o enchimento dos vasos, o herbicida sulfentrazone foi aplicado em pré-emergência, utilizando-se um pulverizador pressurizado com $\mathrm{CO}_{2} \mathrm{e}$ bico XR 110.02, com volume de calda de $200 \mathrm{~L} \mathrm{ha}^{-1}$. As condições climáticas, durante a aplicação, foram: temperatura do ar de $25^{\circ} \mathrm{C}$ e do solo de $28,8^{\circ} \mathrm{C}$, umidade relativa de $56 \%$ (com ausência de vento) e 12,5\% de nuvens no céu. Vinte dias após o término desta etapa, realizou-se a semeadura das espécies vegetais, distribuindo-se seis sementes por vaso, à profundidade de $0,5 \mathrm{~cm}$. Após a emergência das plantas, foi feito desbaste, deixando-se duas plantas por vaso. Todos os vasos foram irrigados diariamente com água potável, mantendo-se o solo em $60 \%$ da capacidade de campo. O valor da capacidade de campo foi determinado em teste preliminar (Casaroli \& Lier 2008) à implantação do experimento, considerando-se taxa de decréscimo do teor de água $|\mathrm{d} \theta / \mathrm{dt}|=0,001 \mathrm{~d}^{-1}$.

Aos 60 dias após a semeadura (DAS), as plantas foram cortadas à altura do coleto, sendo a parte aérea descartada. A seguir, foi realizada uma adubação de plantio nos vasos, com base em nova análise dos solos (por tratamento) e nas necessidades médias da cultura bioindicadora [milheto (Pennisetum glaucum)], a qual foi, imediatamente, semeada, distribuindo-se seis sementes por vaso, à profundidade de $0,5 \mathrm{~cm}$, realizando-se, desta maneira, o bioensaio no próprio vaso. A metodologia de bioensaios foi utilizada por ser considerada adequada à detecção de resíduos do herbicida sulfentrazone no solo (Blanco \& Velini 2005). Após a emergência das plântulas, foi feito o desbaste, deixando-se duas plantas por vaso.

Aos 30 DAS e 60 DAS, foi avaliada a altura de plantas $(\mathrm{cm})$, tomando-se, como base para medição, o meristema apical, e a fitotoxicidade (avaliada visualmente, em \%), atribuindo-se notas de acordo com os sintomas de toxidez na parte aérea das plantas, com base em escala variando de 0 a 100 , para ausência de sintomas e morte da planta, respectivamente (Procópio et al. 2004). Aos 60 DAS, foi determinada a biomassa fresca da parte aérea (g). Após a secagem do material colhido, em estufa de circulação forçada, até massa constante, obteve-se a matéria seca da parte aérea $(\mathrm{g})$.

Os dados foram submetidos à análise de variância e teste F, a 5\%. As médias dos efeitos das interações e dos efeitos principais de plantas promissoras foram comparadas pelo teste Tukey, a 5\%, e o efeito das doses de sulfentrazone foi estudado por meio de regressão, a 5\%. Empregaram-se, para a análise dos dados, os programas SAEG e Sigma Plot 11.0.

\section{RESULTADOS E DISCUSSÃO}

Constatou-se efeito significativo das doses de sulfentrazone (Tabelas 1, 2 e 3) sobre a altura, biomassa fresca e seca da parte aérea e também sobre a percentagem de toxicidade deste herbicida na planta indicadora ( $P$. glaucum), quando em sucessão às diferentes espécies fitorremediadoras e às testemunhas sem cultivo prévio. A altura das plantas e a biomassa fresca e seca da parte aérea de P. glaucum diminuíram com o aumento das doses de sulfentrazone, para as quatro espécies avaliadas. A fitotoxicidade visual, para todas as espécies avaliadas, aumentou com o incremento na dose de sulfentrazone.

$\mathrm{O}$ incremento na dose de sulfentrazone aumentou os sintomas de fitointoxicação sobre P. glaucum. $\mathrm{Na}$ primeira avaliação, realizada aos 30 DAS (Tabela 1), houve 55,3\% de fitotoxicidade, na dose de 200 g i.a. ha ${ }^{-1}$, e $78 \%$ de fitotoxicidade, na dose de 400 g i.a. ha ${ }^{-1}$, quando cultivado após C. juncea. Nestas duas doses, quando $P$. glaucum foi cultivado em sucessão às espécies $C$. cajan, $C$. cajan (anão) e 
Tabela 1. Fitotoxicidade de plantas de P. glaucum cultivadas em sucessão a quatro espécies potencialmente fitorremediadoras e uma testemunha sem cultivo prévio, avaliada aos 30 e 60 dias após a semeadura (DAS), em função de quatro doses de sulfentrazone (São Mateus, ES, 2010).

\begin{tabular}{|c|c|c|c|c|c|}
\hline Dose (g i.a. ha $\left.{ }^{-1}\right)$ & Testemunha & C. cajan & C. cajan (anão) & C. ensiformis & C. juncea \\
\hline & \multicolumn{5}{|c|}{ Fitotoxicidade $(\%)$ aos $30 \mathrm{DAS}$} \\
\hline 0 & $0,0 \mathrm{a}^{(1)}$ & $0,0 \mathrm{a}^{(2)}$ & $0,0 \mathrm{a}^{(3)}$ & $0,0 \mathrm{a}^{(4)}$ & $0,0 \mathrm{a}^{(5)}$ \\
\hline 200 & $93,5 \mathrm{a}$ & $90,0 \mathrm{a}$ & $90,8 \mathrm{a}$ & $90,0 \mathrm{a}$ & $55,3 \mathrm{~b}$ \\
\hline 400 & 96,3 a & $97,3 \mathrm{a}$ & $95,3 \mathrm{a}$ & $96,0 \mathrm{a}$ & $78,0 \mathrm{~b}$ \\
\hline 800 & $100,0 \mathrm{a}$ & $100,0 \mathrm{a}$ & $100,0 \mathrm{a}$ & $100,0 \mathrm{a}$ & $100,0 \mathrm{a}$ \\
\hline \multirow[t]{2}{*}{ C.V. $(\%)$} & 3,05 & & & & \\
\hline & \multicolumn{5}{|c|}{ Fitotoxicidade (\%) aos 60 DAS } \\
\hline 0 & $0,0 \mathrm{a}^{(6)}$ & $0,0 \mathrm{a}^{(7)}$ & $0,0 \mathrm{a}^{(8)}$ & $0,0 \mathrm{a}^{(9)}$ & $0,0 \mathrm{a}^{(10)}$ \\
\hline 200 & $78,8 \mathrm{a}$ & $60,0 \mathrm{c}$ & $68,8 \mathrm{~b}$ & $68,8 \mathrm{~b}$ & $45,0 \mathrm{~d}$ \\
\hline 400 & $96,0 \mathrm{a}$ & $93,3 \mathrm{a}$ & $90,8 \mathrm{a}$ & $96,0 \mathrm{a}$ & $77,0 \mathrm{~b}$ \\
\hline 800 & $100,0 \mathrm{a}$ & $100,0 \mathrm{a}$ & $100,0 \mathrm{a}$ & $100,0 \mathrm{a}$ & $100,0 \mathrm{a}$ \\
\hline C.V. $(\%)$ & 5,74 & & & & \\
\hline
\end{tabular}

C. ensiformis, apresentou sintomas de fitotoxicidade acima de $90 \%$. Na dose de 800 g i.a. ha ${ }^{-1}$, C. juncea, assim como as demais espécies, não promoveu suficiente descontaminação do solo, para que P. glaucum sobrevivesse, evidenciando a alta sensibilidade desta espécie ao sulfentrazone (Dan et al. 2011) e reforçando o cuidado com a semeadura desta cultura, em áreas com histórico de aplicações deste herbicida.

Quando foi realizada a segunda avaliação, aos 60 DAS (Tabela 1), os sintomas de fitotoxicidade de P. glaucum, na dose de 200 g i.a. ha ${ }^{-1}$, foram menores, em valores absolutos, para todas as espécies avaliadas, quando comparados à avaliação aos 30 DAS. Todavia, os sintomas visuais de intoxicação ainda permaneceram elevados, exceto quando cultivado após $C$. juncea ( $45 \%$ de fitotoxicidade). Na dose de 400 g i.a. ha ${ }^{-1}$, apenas $C$. juncea diferiu da testemunha.

O germinação inicial de C. juncea é do tipo epígea, sendo que os hipocótilos se levantam do substrato, levando os cotilédones para fora, sua radícula assume rapidamente caráter apical, com raízes secundárias finas, e os protófilos assumem formato aculeado, podendo-se inferir, desta maneira, o surgimento de uma raiz mais profunda e agressiva. Estas características são importantes para uma planta remediadora, pois, normalmente, estão relacionadas a uma maior capacidade de absorção do herbicida pelas raízes (Newman et al. 1998). Outro aspecto a ser considerado é que a maioria dos compostos orgânicos passa por transformações nas células das plantas, antes de serem isolados em vacúolos ou ligarem-se a estruturas celulares insolúveis, como a lignina (Macek et al. 2000).
$\mathrm{O}$ alto teor de fibras de C. juncea, incluindo a lignina (Carvalho et al. 2010), pode ter favorecido este mecanismo de fitorremediação. Além deste mecanismo, de forma adicional, a C. juncea pode, ainda, promover a remediação pela rizosfera, pois flavonoides e outros compostos liberados pelas raízes podem estimular o crescimento e a atividade de micro-organismos degradadores ou, ainda, reagir com o herbicida, imobilizando-o (Ferro et al. 1999, Leigh et al. $2002 \mathrm{e}$ 2006, Chaudhry et al. 2005). Além disto, o crescimento e morte das raízes promove a aeração do solo, o que pode aumentar a degradação oxidativa de compostos orgânicos (Leigh et al. 2002, Kuiper et al. 2004).

Para as características altura de plantas e biomassa fresca e seca, as espécies fitorremediadoras apresentaram comportamento similar. Quando cultivado em sucessão a praticamente todas as espécies avaliadas, P. glaucum apresentou brusca redução na altura e biomassa fresca e seca, a partir da dose de $200 \mathrm{~g}$ i.a. ha ${ }^{-1}$, não se desenvolvendo na dose de 800 g i.a. ha ${ }^{-1}$, em razão da elevada toxicidade apresentada pelo sulfentrazone (Tabelas 2 e 3 ). Percebe-se, também, que a menor redução na altura e biomassa fresca e seca ocorreu quando P. glaucum foi antecedido por $C$. juncea.

Belo et al. (2011) selecionaram Helianthus annus como a espécie mais eficiente na remediação de solos contaminados com sulfentrazone. As espécies C. ensiformis, Dolichos lab lab e Arachis hypogaea também apresentaram potencial fitorremediador, em relação a este herbicida, porém, ainda não há relatos na literatura sobre a capacidade remediadora de $C$. juncea, quanto ao sulfentrazone. 
Santos et al. (2004a) observaram que, quando as espécies C. ensiformis, Mucuna aterrima e Raphanus sativus foram cultivadas em solos contaminados com o herbicida trifloxysulfuron sodium, não houve efeito sobre o milho cultivado em sequência. Desta forma, os autores concluíram que estas espécies são eficientes na remediação desta molécula.

Observou-se (Tabela 2) maior altura das plantas de $P$. glaucum, quando cultivadas em sucessão a C. juncea, nas doses de 200 g i.a. ha ${ }^{-1}$ e 400 g i.a. ha ${ }^{-1}$, em ambas as avaliações, entretanto, no solo tratado com 400 g i.a. ha-1 de sulfentrazone, a redução na altura de $P$. glaucum foi mais acentuada. Percebe-se que, na dose de $200 \mathrm{~g}$ i.a. ha-1 ${ }^{-1}$, na primeira avaliação, na qual o solo foi cultivado com $C$. cajan, a redução na altura foi menor, quando comparada à testemunha sem cultivo e àquela cultivada após as espécies $C$. cajan (anão) e C. ensiformis. Contudo, na segunda avaliação, P. glaucum, cultivado em sucessão a C. cajan (anão) e C. ensiformis, exibiu certa recuperação, apresentando médias superiores à testemunha e C. cajan. Isto indica que estas duas espécies estão remediando o solo contaminado com sulfentrazone, mesmo que em proporções inferiores às de $C$. juncea. Também é possível concluir que, à medida em que as plantas crescem, sua ação fitorremediadora é incrementada, notadamente pela maior capacidade de absorção (Procópio et al. 2008).

Para a biomassa fresca e seca das plantas de P. glaucum (Tabela 3), o comportamento foi similar ao observado para a altura. Houve incremento da biomassa, quando $C$. juncea foi cultivada anteriormente a $P$. glaucum, possibilitando o seu desenvolvimento

Tabela 2. Altura das plantas de P. glaucum cultivadas em sucessão a quatro espécies potencialmente fitorremediadoras e uma testemunha sem cultivo prévio, avaliada aos 30 e 60 dias após a semeadura (DAS), em função de quatro doses de sulfentrazone (São Mateus, ES, 2010).

\begin{tabular}{|c|c|c|c|c|c|}
\hline Dose (g i.a. ha $\left.{ }^{-1}\right)$ & Testemunha & C. cajan & C. cajan (anão) & C. ensiformis & C. juncea \\
\hline & \multicolumn{5}{|c|}{ Altura das plantas $(\mathrm{cm})$ aos 30 DAS } \\
\hline 0 & $41,7 \mathrm{c}^{(1)}$ & $52,0 \mathrm{a}^{(2)}$ & $50,3 \mathrm{ab}^{(3)}$ & $47,7 \mathrm{ab}^{(4)}$ & $46,7 \mathrm{bc}^{(5)}$ \\
\hline 200 & $10,0 \mathrm{c}$ & $17,7 \mathrm{~b}$ & $13,0 \mathrm{bc}$ & $13,1 \mathrm{bc}$ & $24,1 \mathrm{a}$ \\
\hline 400 & $3,5 \mathrm{~b}$ & $4,9 \mathrm{~b}$ & $5,4 \mathrm{~b}$ & $5,3 \mathrm{~b}$ & $11,0 \mathrm{a}$ \\
\hline 800 & $0,0 \mathrm{a}$ & $0,0 \mathrm{a}$ & $0,0 \mathrm{a}$ & $0,0 \mathrm{a}$ & $0,0 \mathrm{a}$ \\
\hline \multirow{2}{*}{ C.V. $(\%)$} & 15,26 & & & & \\
\hline & \multicolumn{5}{|c|}{ Altura das plantas $(\mathrm{cm})$ aos 60 DAS } \\
\hline 0 & $118,5 b^{(6)}$ & $139,9 a^{(7)}$ & $122,3 \mathrm{~b}^{(8)}$ & $143,8 \mathrm{a}^{(9)}$ & $135,6 \mathrm{a}^{(10)}$ \\
\hline 200 & $19,6 \mathrm{c}$ & $25,9 \mathrm{c}$ & $38,5 \mathrm{~b}$ & $42,2 \mathrm{~b}$ & $58,2 \mathrm{a}$ \\
\hline 400 & $7,8 \mathrm{~b}$ & $10,8 \mathrm{~b}$ & $15,5 \mathrm{~b}$ & $9,5 \mathrm{~b}$ & $28,8 \mathrm{a}$ \\
\hline 800 & $0,0 \mathrm{a}$ & $0,0 \mathrm{a}$ & $0,0 \mathrm{a}$ & $0,0 \mathrm{a}$ & $0,0 \mathrm{a}$ \\
\hline C.V. $(\%)$ & 11,25 & & & & \\
\hline
\end{tabular}

Médias não seguidas de mesma letra, na linha, diferem pelo teste Tukey, a $5 \%{ }^{(1)} \hat{\mathrm{Y}}=41,5979 * \mathrm{e}^{(-0,0068899 * \mathrm{X})}, \mathrm{R}^{2}=0,97 ;{ }^{(2)} \hat{\mathrm{Y}}=52,0999 * \mathrm{e}^{(-0,0055677 * \mathrm{X})}$, $\mathrm{R}^{2}=0,99 ;{ }^{(3)} \hat{\mathrm{Y}}=50,1338^{*} \mathrm{e}^{\left(-0,0064351^{*} \mathrm{X}\right)}, \mathrm{R}^{2}=0,99 ;{ }^{(4)} \hat{\mathrm{Y}}=47,5871^{*} \mathrm{e}^{\left(-0,0061734^{*} \mathrm{X}\right)}, \mathrm{R}^{2}=0,99 ;{ }^{(5)} \hat{\mathrm{Y}}=47,1072^{*} \mathrm{e}^{\left(-0,0036288^{*} \mathrm{X}\right)}, \mathrm{R}^{2}=0,97 ;{ }^{(6)} \hat{\mathrm{Y}}=118,371^{*} \mathrm{e}^{\left(-0,00862924^{*} \mathrm{X}\right)}$, $\mathrm{R}^{2}=0,99 ;{ }^{(7)} \hat{\mathrm{Y}}=139,679^{*} \mathrm{e}^{(-0,00804393 * \mathrm{X})}, \mathrm{R}^{2}=0,99 ;{ }^{(8)} \hat{\mathrm{Y}}=122,060^{*} \mathrm{e}^{\left(-0,00558126^{*} \mathrm{X}\right)}, \mathrm{R}^{2}=0,99 ;{ }^{(9)} \hat{\mathrm{Y}}=143,966^{*} \mathrm{e}^{\left(-0,00629083^{*} \mathrm{X}\right)}, \mathrm{R}^{2}=0,99 ;{ }^{(10)} \hat{\mathrm{Y}}=135,558^{*} \mathrm{e}^{\left(-0,00414638^{*} \mathrm{X}\right)}, \mathrm{R}^{2}=0,99$

Tabela 3. Biomassa fresca e seca da parte aérea de plantas de $P$. glaucum cultivadas em sucessão a quatro espécies potencialmente fitorremediadoras e uma testemunha sem cultivo prévio, em função de quatro doses de sulfentrazone (São Mateus, ES, 2010).

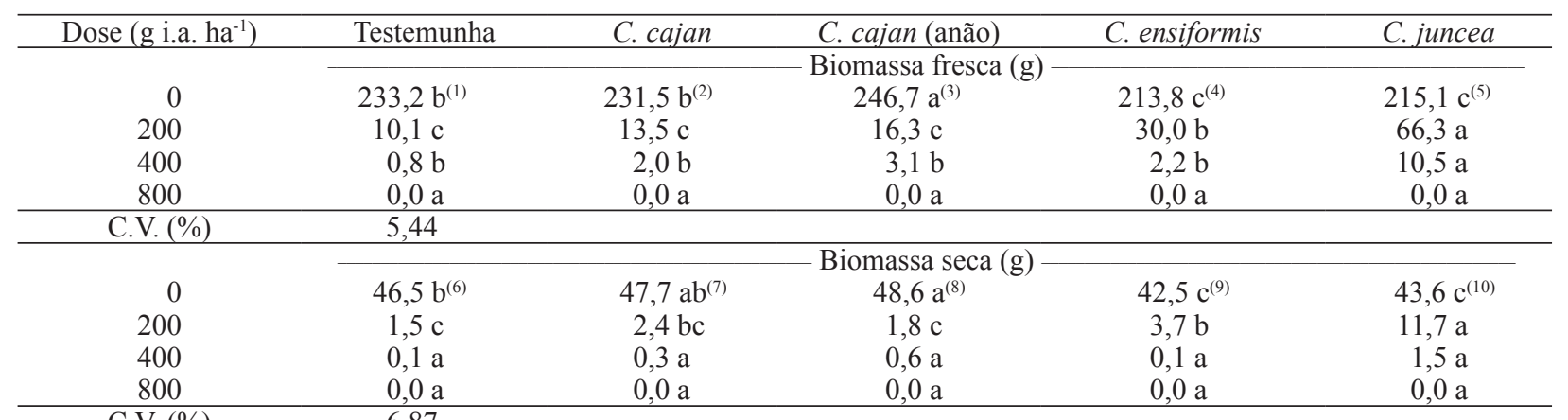

Médias não seguidas de mesma letra, na linha, diferem pelo teste Tukey, a 5\% . ${ }^{(1)} \hat{\mathrm{Y}}=233,249^{*} \mathrm{e}^{\left(-0,0156808^{*} \mathrm{X}\right)}, \mathrm{R}^{2}=0,99 ;{ }^{(2)} \hat{\mathrm{Y}}=231,506^{*} \mathrm{e}^{\left(-0,0141702^{*} \mathrm{X}\right)}, \mathrm{R}^{2}=0,99$;

(3) $\hat{\mathrm{Y}}=246,676^{*} \mathrm{e}^{\left(-0,0135153^{*} \mathrm{X}\right)}, \mathrm{R}^{2}=0,99 ;{ }^{(4)} \hat{\mathrm{Y}}=213,797 * \mathrm{e}^{\left(-0,00990281^{*} \mathrm{X}\right)}, \mathrm{R}^{2}=0,99 ;{ }^{(5)} \hat{\mathrm{Y}}=215,684^{*} \mathrm{e}^{\left(-0,00623973^{*} \mathrm{X}\right)}, \mathrm{R}^{2}=0,99 ;{ }^{(6)} \hat{\mathrm{Y}}=46,5124^{*} \mathrm{e}^{\left(-0,017113^{*} \mathrm{X}\right)}, \mathrm{R}^{2}=0,99$;

(7) $\hat{Y}=47,7346^{*} \mathrm{e}^{\left(-0,0148727^{*} \mathrm{X}\right)}, \mathrm{R}^{2}=0,99 ;{ }^{(8)} \hat{\mathrm{Y}}=48,5593^{*} \mathrm{e}^{\left(-0,0165046^{*} \mathrm{X}\right)}, \mathrm{R}^{2}=0,99 ;{ }^{(9)} \hat{\mathrm{Y}}=42,5135^{*} \mathrm{e}^{\left(-0,012202 *^{*} \mathrm{X}\right)}, \mathrm{R}^{2}=0,99 ;{ }^{(10)} \hat{\mathrm{Y}}=43,6762^{*} \mathrm{e}^{\left(-0,00689409^{*} \mathrm{X}\right)}, \mathrm{R}^{2}=0,99$. 
em solo com este herbicida, com menor redução de biomassa fresca até a dose de $400 \mathrm{~g}$ i.a. ha ${ }^{-1}$ e de biomassa seca até a dose de 200 g i.a. ha ${ }^{-1}$.

Pires et al. (2008), avaliando a fitorremediação de solo contaminado com diferentes níveis de tebuthiuron, utilizando $C$. juncea como espécie indicadora da presença deste herbicida, verificaram que as espécies $C$. ensiformis e $C$. cajan apresentaram os melhores desempenhos, sendo indicadas para fitorremediação de solos, tendo recebido até 1.000 g i.a. ha ${ }^{-1}$ de tebuthiuron.

Santos et al. (2004a) selecionaram C. ensiformis como uma das mais eficientes espécies na fitorremediação do herbicida trifloxysulfuron sodium. Todavia, para o presente trabalho, estas espécies mostraram-se menos eficientes na fitorremediação de solos contaminados com sulfentrazone, utilizando-se $P$. glaucum como espécie indicadora da presença deste herbicida, quando comparadas a $C$. juncea. Possivelmente, isto ocorre devido ao mecanismo de tolerância destas espécies ao sulfentrazone. Elas podem se valer de um mecanismo com o qual simplesmente reduzem a absorção do herbicida e produzem baixa quantidade de exsudatos pelas raízes, fazendo com que a maior parte do sulfentrazone permaneça no solo, sem sofrer alterações em sua estrutura.

Algumas respostas que buscam elucidar a fitorremediação foram obtidas por Merini et al. (2009), os quais atestaram a eficiência de Lolium multiflorum na fitorremediação do herbicida atrazine. Estes autores observam que L. multiflorum apresenta alta taxa de degradação inicial do atrazine e sua tolerância a este herbicida deve-se ao aumento do seu metabolismo, por meio da P450. Também Sulmon et al. (2007) verificaram que o aumento do teor de sacarose na espécie Arabidopsis thaliana pode aumentar a tolerância e a fitodegradação do atrazine por esta espécie. Já Kawahigashi et al. (2006) estudaram a capacidade fitorremediadora de plantas transgênicas de arroz e concluíram que estas reduziram, em níveis consideráveis, os herbicidas metolachlor e atrazine no solo.

\section{CONCLUSÃO}

C. juncea foi a espécie que apresentou a maior capacidade de fitorremediar solos contaminados com o herbicida sulfentrazone, até a dose de 400 gi.a. ha ${ }^{-1}$. C. cajan (anão) e C. ensiformis também apresentaram potencial remediador do solo, porém, em níveis inferiores a $C$. juncea.

\section{AGRADECIMENTOS}

Ao Conselho Nacional de Desenvolvimento Científico e Tecnológico ( $\mathrm{CNPq}$ ), pelo financiamento do projeto e concessão de bolsas.

\section{REFERÊNCIAS}

ANDERSON, T. A.; COATS, J. R. Screening rhizosphere soil samples for the ability to mineralize elevated concentrations of atrazine and metolachlor. Journal of Environmental Science and Health, New York, v. 30, n. 4, p. 473-484, 1995.

ARTHUR, E. L. et al. Degradation of an atrazine and metolachlor herbicide mixture in pesticide-contaminated soils from two agrochemical dealerships in Iowa. Water, Air, and Soil Pollution, Dordrecht, v. 119, n. 1-4, p. 7590, 2000.

BELO, A. F. et al. Potencial de espécies vegetais na remediação de solo contaminado com sulfentrazone. Planta daninha, Viçosa, v. 29, n. 4, p. 821-828, 2011.

BLANCO, F. M. G.; VELINI, E. D.; BATISTA FILHO, A. Persistência do herbicida sulfentrazone em solo cultivado com cana-de-açúcar. Bragantia, Campinas, v. 69, n. 1, p. 71-75, 2010.

BLANCO, F. M. G.; VELINI, E. D. Persistência do herbicida sulfentrazone em solo cultivado com soja e seu efeito em culturas sucedâneas. Planta Daninha, Viçosa, v. 23, n. 4, p. 693-700, 2005.

CARVALHO, A. M. et al. Teores de hemiceluloses, celulose e lignina em plantas de cobertura com potencial para sistema plantio direto no Cerrado. Planaltina: Embrapa Cerrados, 2010. (Boletim de pesquisa e desenvolvimento, 290).

CASAROLI, D.; LIER, Q. J. V. Critérios para determinação da capacidade de vaso. Revista Brasileira de Ciência do Solo, Viçosa, v. 32, n. 1, p. 59-66, 2008.

CHAUDHRY, Q. et al. Utilizing the synergy between plants and rhizosphere microorganisms to enhance breakdown of organic pollutants in the environment. Environmental Science and Pollution Research, Berlin, v. 12, n. 1, p. 34-48, 2005.

COMPANHIA NACIONAL DE ABASTECIMENTO (Conab). Acompanhamento de safra brasileira: cana-deaçúcar, segundo levantamento, agosto/2012. Brasília, DF: Conab, 2012.

DAN, H. A. et al. Atividade residual de herbicidas préemergentes aplicados na cultura da soja sobre o milheto cultivado em sucessão. Planta Daninha, Viçosa, v. 29, n. 2, p. 437-445, 2011. 
EMPRESA, BRASILEIRA DE PESQUISA AGROPECUÁRIA (Embrapa). Manual de análises de solo. 2. ed. Rio de Janeiro: Embrapa Solos, 1997.

EMPRESA BRASILEIRA DE PESQUISA AGROPECUÁRIA (Embrapa). Manual de análises químicas de solos, plantas e fertilizantes. Brasília, DF: Embrapa Comunicação para Transferência de Tecnologia, 1999.

EMPRESA BRASILEIRA DE PESQUISA AGROPECUÁRIA (Embrapa). Sistema brasileiro de classificação de solos. 2. ed. Rio de Janeiro: Embrapa Solos, 2006.

FERRO, A. M. et al. Phytoremediation of soils contaminated with wood preservatives: greenhouse and field evaluations. International Journal of Phytoremediation, Boca Raton, v. 1, n. 3, p. 289-306, 1999.

KAWAHIGASHI, H. et al. Phytoremediation of the herbicides atrazine and metolachlor by transgenic rice plants expressing human CYP1A1, CYP2B6, and CYP2C19. Journal of Agricultural and Food Chemistry, Washington, DC, v. 54, n. 8, p. 2985-2991, 2006.

KUIPER, I. et al. Rhizoremediation: a beneficial plant-microbe interaction. Molecular Plant-Microbe Interactions, St. Paul, v. 17, n. 1, p. 6-15, 2004.

LEIGH, M. B. et al. Polychlorinated biphenyl (PCB)degrading bacteria associated with trees in a PCBcontaminated site. Applied and Environmental Microbiology, Washington, DC, v. 72, n. 4, p. 2331-2342, 2006.

LEIGH, M. B. et al. Root turnover: an important source of microbial substrates in rhizosphere remediation of recalcitrant contaminants. Environmental Science \& Technology, Easton, v. 36, n. 7, p. 1579-1583, 2002.

MACEK, T.; MACKOVÁ, M.; KÁS, J. Exploitation of plants for the removal of organics in environmental remediation. Biotechnology Advances, New York, v. 18, n. 1, p. 23-34, 2000.

MADALÃO, J. C. Seleção de espécies para a fitorremediação de solos contaminados com o herbicida sulfentrazone. 2011. 103 f. Dissertação (Mestrado em Produção Vegetal) - Universidade Federal do Espírito Santo, Alegre, 2011.

MELO, C. A. D. et al. Efeito residual de sulfentrazone, isoxaflutole e oxyfluorfen em três solos. Planta Daninha, Viçosa, v. 28, n. 4, p. 835-842, 2010.

MERINI, L. J. et al. Phytoremediation potential of the novel atrazine tolerant Lolium multiflorum and studies on the mechanisms involved. Environmental Pollution, Barking, v. 157, n. 11, p. 3059-3063, 2009.

MONQUERO, P. A. et al. Lixiviação e persistência dos herbicidas sulfentrazone e Imazapic. Planta Daninha, Viçosa, v. 28, n. 1, p. 185-195, 2010.

NEWMAN, L. A. et al. Phytoremediation of organic contaminants: a review of phytoremediation research at the University of Washington. Journal of Soil Contamination, Boca Raton, v. 7, n. 4, p. 531-542, 1998.
PIRES, F. R.; EGREJA FILHO, F. B.; PROCÓPIO, S. O. Inferências sobre mineralogia dos solos, sorção e fitorremediação de herbicidas. In: FERREIRA, A. et al. (Orgs.). Tópicos especiais em produção vegetal I. Vitória: Edufes, 2009. p. 391-406.

PIRES, F. R. et al. Adubos verdes na fitorremediação de solos contaminados com o herbicida tebuthiuron. Caatinga, Mossoró, v. 19, n. 1, p. 92-97, 2006.

PIRES, F. R. et al. Avaliação da fitorremediação de tebuthiuron utilizando Crotalaria juncea como planta indicadora. Revista Ciência Agronômica, Fortaleza, v. 39, n. 2, p. 245-250, 2008.

PIRES, F. R. et al. Fitorremediação de solos contaminados com tebuthiuron utilizando-se espécies cultivadas para adubação verde. Planta Daninha, Viçosa, v. 23, n. 4, p. 711-717, 2005b.

PIRES, F. R. et al. Inferências sobre atividade rizosférica de espécies com potencial para fitorremediação do herbicida tebuthiuron. Revista Brasileira de Ciência do Solo, Viçosa, v. 29, n. 4, p. 627-634, 2005 a.

PROCÓPIO, S. O. et al. Development of bean plants in soil contaminated with trifloxysulfuron sodium after Stizolobium aterrimum and Canavalia ensiformis cultivation. Planta Daninha, Viçosa, v. 25, n. 1, p. 87-96, 2007.

PROCÓPIO, S. O. et al. Fitorremediação de solo contaminado com picloram por capim-pé-de-galinhagigante (Eleusine coracana). Revista Brasileira de Ciência do Solo, Viçosa, v. 32, n. 6, p. 2517-2523, 2008.

PROCÓPIO, S. O. et al. Seleção de plantas com potencial para fitorremediação de solos contaminados com o herbicida trifloxysulfuron sodium. Planta Daninha, Viçosa, v. 22, n. 2, p. 315-322, 2004.

SANTOS, J. B. et al. Fitorremediação do herbicida trifloxysulfuron sodium. Planta Daninha, Viçosa, v. 22, n. 2, p. 223-330, 2004a.

SANTOS, J. B. et al. Seletividade do herbicida trifloxysulfuron sodium para fins de fitorremediação. Revista Ceres, Viçosa, v. 51, n. 293, p. 129-141, 2004 b.

SULMON, C. et al. Sucrose amendment enhances phytoaccumulation of the herbicide atrazine in Arabidopsis thaliana. Environmental Pollution, Barking, v. 145, n. 2, p. 507-515, 2007.

UNIÃO DA INDÚSTRIA DA CANA-DE-AÇÚCAR (Unica). Cana-de-açúcar no Brasil. 2010. Disponível em: <http://www.unica.com.br/FAQ>. Acesso em: 09 out. 2012.

VIVIAN, R. et al. Persistência de sulfentrazone em Argissolo Vermelho-Amarelo cultivado com cana-deaçúcar. Planta Daninha, Viçosa, v. 24, n. 4, p. 741-750, 2006.

WILSON, P. C.; WHITWELL, T.; KLAINE, S. J. Phytotoxicity, uptake, and distribution of ${ }^{14} \mathrm{C}$-simazine in Acorus gramenius and Pontederia cordata. Weed Science, Champaign, v. 48, n. 6, p. 701-709, 2000. 